\title{
Imaging spectroscopy of planetary surfaces: Improving the spatial contrast
}

\begin{abstract}
G. Bellucci
Received December 29, 1997; accepted May 15, 1998

Abstract. A method to improve the spatial contrast of planetary images acquired by means of push-broom imaging spectrometers is presented. The technique allows to better recognize the boundaries of spatially coherent units with different mineralogic composition. We have analyzed the case of a surface observed by means of an imaging spectrometer from a ground-based telescope. It is assumed that image degradation is mainly due to the atmosphere placed between the surface remotely sensed and the instrumentation. A possible extension to space born imaging spectrometers affected by random errors of the pointing system is also discussed. An example relative to the observation of the Moon is given.
\end{abstract}

CNR Istituto di Fisica dello Spazio Interplanetario Area di Ricerca di Tor Vergata Via Fosso del Cavaliere, 00133 Rome, Italy

Mapping Spectrometer, designed for the CRAF (Comet Randevouz Asteroid Flyby) mission (Juergens et al. 1990) but now intended to observe the Saturnian system of rings and satellites from aboard the U.S. Cassini spacecraft. When solar radiation interacts with a planetary surface, the reflected component contains absorption bands which are diagnostic of the mineralogic composition of the surface (Adams 1974). An imaging spectrometer acquires many images in several $(>100)$ narrow spectral bands, thus providing the spectrum of each pixel in the scene. Combining spectroscopy and imaging together is then possible to generate compositional maps of large areas. If an atmosphere is present between the surface and the instru-
Key words: instrumentation: spectrographs — techniques: spectroscopic — planets and satellites: general — space vehicles - methods: statistical

\section{Introduction}

Imaging spectroscopy is a relatively new technique in remote sensing which has become available in electronic form thanks to new matrix solid state detectors and improvements in optical technology. Imaging spectrometers have been recognized to be powerful instruments for the remote sensing of solar system bodies and have been included, as part of the payload, in many planetary missions. Electronic imaging spectrometers were firstly used for terrestrial remote sensing (Vane et al. 1984). The first spaceborne instruments designed for planetary observation were the Galileo Near - Infrared Mapping Spectrometer (NIMS), launched aboard the U.S. Galileo mission on 1990 with the aim to study the geochemistry of Jovian satellites and the atmosphere of Jupiter (Aptaker 1987) and the Phobos Infrared Spectrometer (ISM), flown on the Soviet Phobos space probe on 1988 (Bibring et al. 1989). To date, an other instrument has been developed for planetary exploration: the VIMS, Visible Near Infrared ment, the air turbulence degrades every monochromatic image, lowering the signal to noise ratio and worsening the spatial resolution. Moreover, in the case of push-broom imaging spectrometers which form a bidimensional image by scanning the slit across the planetary surface and acquiring the spectra of the regions imaged by the slit, the scanning mechanisms introduce an error caused by the noise in the driver electronics and tolerances in the mechanics. All these effects contribute to the degradation of imaging performance. These problems are not present in Fourier transform imaging spectrometers which do not have scanning elements even if pointing errors can still be present. Moreover, push-broom imaging spectrometers have an intrinsic limitation in the maximum achievable spatial resolution. This parameter, in fact, competes with the maximum achievable signal to noise ratio. Owing to the fixed scanning velocity, high spatial resolution images require relatively low integration times yielding, consequently, low signal to noise data. In the first section of this paper, the basic concepts of the methodology used to increase the content of spatial information of imaging spectroscopy data is discussed. The details are illustrated in the second part. The rest of the paper reports some results of the method applied on imaging spectroscopy data of the Moon. The last section discusses the possible extension of the technique to planetary remote sensing from space vehicles. 


\section{Spatial contrast enhancement: General concepts}

Changes of pressure and temperature in the atmosphere cause changes in its density and refractive index (Goodman 1985). These, in turn, if they are inhomogeneous, result in the distortion of the wavefront passing through the atmosphere from any object point. Such changes are always present in the natural atmosphere. They vary randomly and are called turbulence. As a consequence of turbulence, an image formed through the atmosphere will, in general exhibit scintillation, motion, and blurring effects. They pose an ultimate limit on the image quality attainable through the atmosphere. In astronomical observation this effect is called seeing and represents the major limitation on the resolution and geometrical accuracy. The effect of atmospheric turbulence is a spatial filtering of the image, which is smeared by the loss of spatial coherence over the pupil plane (Fried 1966). A full interpretation of images is then possible if the system response (point spread function or PSF) is known. Iterative deconvolution techniques have been developed (maximum entropy, clean and Lucy, Hanish \& White 1993) which aim to estimate the ideal image that would be observed if no image degradation were present. From this point of view, full restoration of the spatial details acquired by means of a push-broom imaging spectrometer is not possible, or at least, very difficult. The reason is due to the fact that the lines forming an image are acquired at different times. The system response along the slit can be known by taking the image line of a point source. However, due to the optical aberrations, it is a function of position along the slit. The across slit response is unknown, due to the random nature of the atmospheric turbulence. The method we are presenting here is an image sharpening procedure which improves the spatial content of an image by utilizing spectral correlations among adjacent pixels. From the previous discussion we have seen how the atmosphere causes the blurring of an image: photons coming from the instantaneous field of view pertaining a certain pixel, are spread over a larger number of picture elements. A planetary surface is never composed of a single uniform material, and therefore the spatial blurring also causes mixing of mineralogically different regions of the surface. This situation is illustrated in Fig. 1, where two spectrally distinct units are indicated by letters A and B (Fig. 1a). At the A-B border, blurring causes mixing of $\mathrm{A}$ and $\mathrm{B}$ spectra and generates a third zone $(\mathrm{C})$ having a spectrum intermediate between the A and B spectra (Fig. 1b). Image sharpening can then be accomplished by means of spectral unmixing procedures. Note that spectral unmixing is particularly effective with imaging spectroscopy data, because the spectrum of each pixel is defined with high accuracy, due to the great number of bands. In planetary multiband imaging (generally less than 10 spectral bands), the pixel spectrum is less detailed and spectral unmixing is then less effective. If the scale of mixing is large (as in planetary remote sensing), mixing generally occurs in a linear fashion while for microscopic or intimate mixtures, the mixing is generally not linear (Singer \& McCord 1979). Spectral unmixing is usually done by using known endmembers, seeking to derive the apparent fractional abundance of each endmember material in each pixel, given a set of known or assumed spectral endmembers (Adams et al. 1986). These known endmembers can be drawn from the data (averages of regions picked using previous knowledge), drawn from a library of pure materials by interactively browsing through the imaging spectrometer data to determine what pure materials exist in the image, or determined using expert systems to identify materials. The mixing endmembers matrix is made up of spectra from the image or a reference library. The problem can be cast in terms of a linear least squares problem. The mixing matrix is inverted and multiplied by the observed spectra to get least-squares estimates of the unknown endmember abundance fractions. Constrains can be placed on the solutions to give positive fractions that sum to unity. Shade and shadow are included either implicitly (fraction sum to 1 or less) or explicitly as an endmember (fractions sum to 1). A possible drawback of this method is that the image endmembers may themselves represent mixtures rather than true compositional endmembers. For example, on Moon this happens at proximity of craters where the materials resulting from an impact event are mixed with preexisting soils. A simple measure of pixel spectral homogeneity is to reduce the least squares error to a significant level through a trial-and-error method, by continually refine the endmember selection. However, this procedure can be very time consuming, specially when the spectra to process are composed of many bands. For this reason, we adopted a solution based on the use of another linear technique, the Principal Component Analysis (henceforth PCA). This technique is very sensitive to the spectral content of the data, and can then be used for spectral unmixing.

\section{Description of the method}

PCA is a technique generally used to enhance the information content of multiband data and to reduce the dimensionality of the data set (Davis 1986; Chevrel et al. 1994; Erard et al. 1991; Jaumann 1991). PCA uses a linear transformation of the data to translate and rotate them in a new coordinate system that maximizes the variance. The rows of the transformation matrix $\mathbf{A}$ are composed of the eigenvectors of the covariance matrix $K_{x}$ between the bands. The matrix A performs a diagonalization of the covariance matrix $K_{x}$ such that the covariance matrix of the transformed imagery

$K_{y}=\mathbf{A} K_{x} \mathbf{A}^{\mathbf{T}}=\mathbf{\Lambda}$

is a diagonal matrix whose elements are the eigenvalues of $K_{x}$ arranged in descending value. The principal 
Table 1. Statistics of three principal components of the image cube discussed in the text. The units are in digital numbers

\begin{tabular}{cccccc}
\hline PC Image & Min & Max & Mean & Stdev & Eigenval \\
\hline 1 & 0.3299 & 2.0179 & 1.0439 & 0.1397 & 1.5817 \\
2 & 0.3253 & 2.0252 & 1.0422 & 0.1407 & 0.0078 \\
3 & 0.3287 & 2.0364 & 1.0442 & 0.1417 & 0.0029 \\
\hline
\end{tabular}
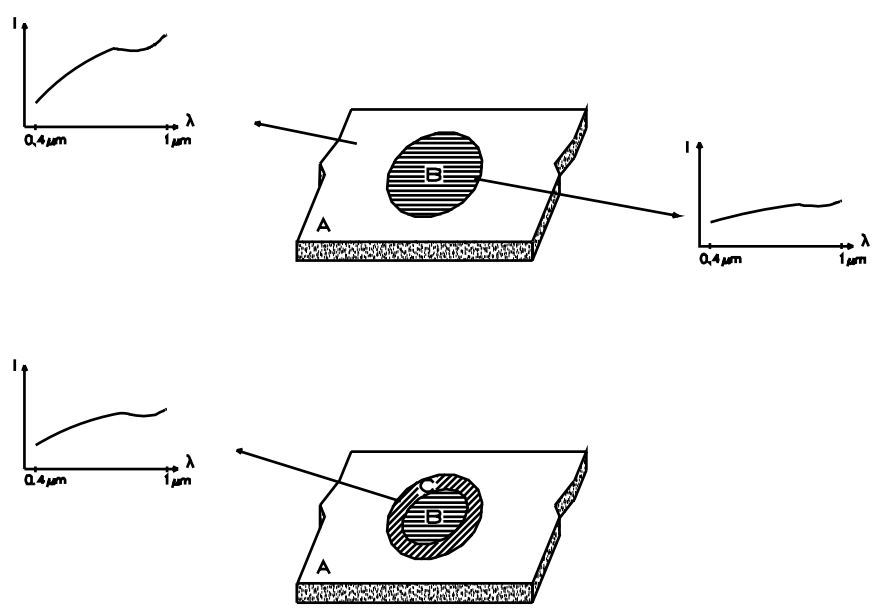

Fig. 1. Sketch illustrating the sharpening method. a) Two distinct spectral units without spatial blurring. b) The effect of blurring is to produce a third spectral unit (C) and to blur the edges

components decomposition therefore results in a set of decorrelated data planes and the information is contained in few PC images (the eigenvectors) while most of the noise is segregated in the others components. The first transformed image is approximately an albedo picture and depicts the average brightness of the surface while the others components contain the color information. These higher order components are used for our purposes. We applied this technique to an image cube of the Moon composed of $128 \times 258$ spectra of 96 bands each, obtained by means of a visible-near infrared imaging spectrometer $(0.4-1.0 \mu \mathrm{m}$ spectral range, $\Delta \lambda=7.5 \mathrm{~nm})$. Details about the instrumentation can be found in Bellucci et al. 1998 . The PC transformation has not been directly applied to the raw image cube but to a relative image cube. Each spectrum of the original data set, has been normalized respect to an average spectrum of a small area $(10 \times 10$ pixels) chosen within the image. This operation removes at the same time the instrumental and atmospheric transfer functions and allows to enhance spectral differences proper of the lunar surface only. Table 1 summarizes the relevant statistics of $3 \mathrm{PC}$ images which contain most of the image cube variance. Eigenvalues for bands that contain some information are larger than those that contain only noise. The corresponding PC images are spatially coherent, while the noise images do not contain any spatial information.

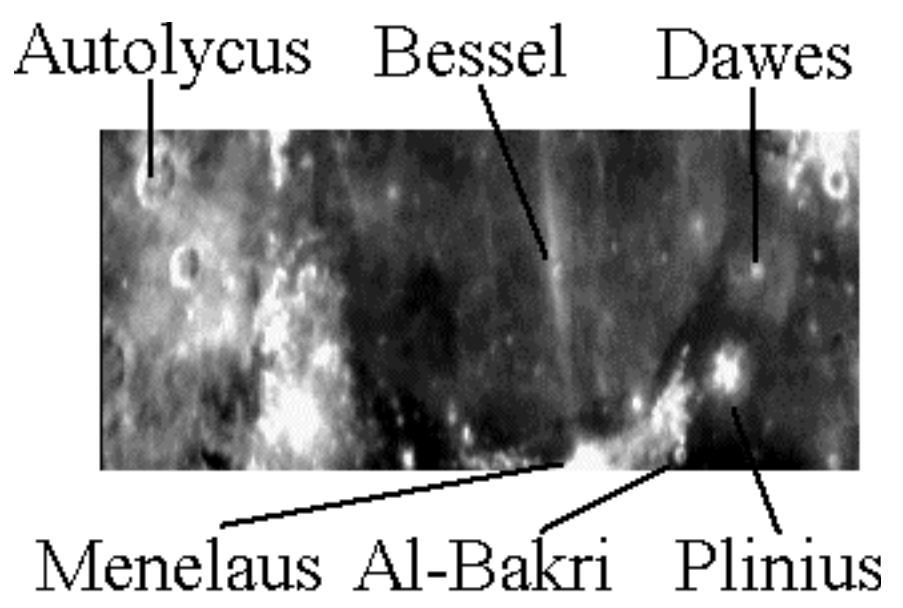

Fig. 2. Raw image of the study region at $0.7 \mu \mathrm{m}$. Main geologic features are indicated
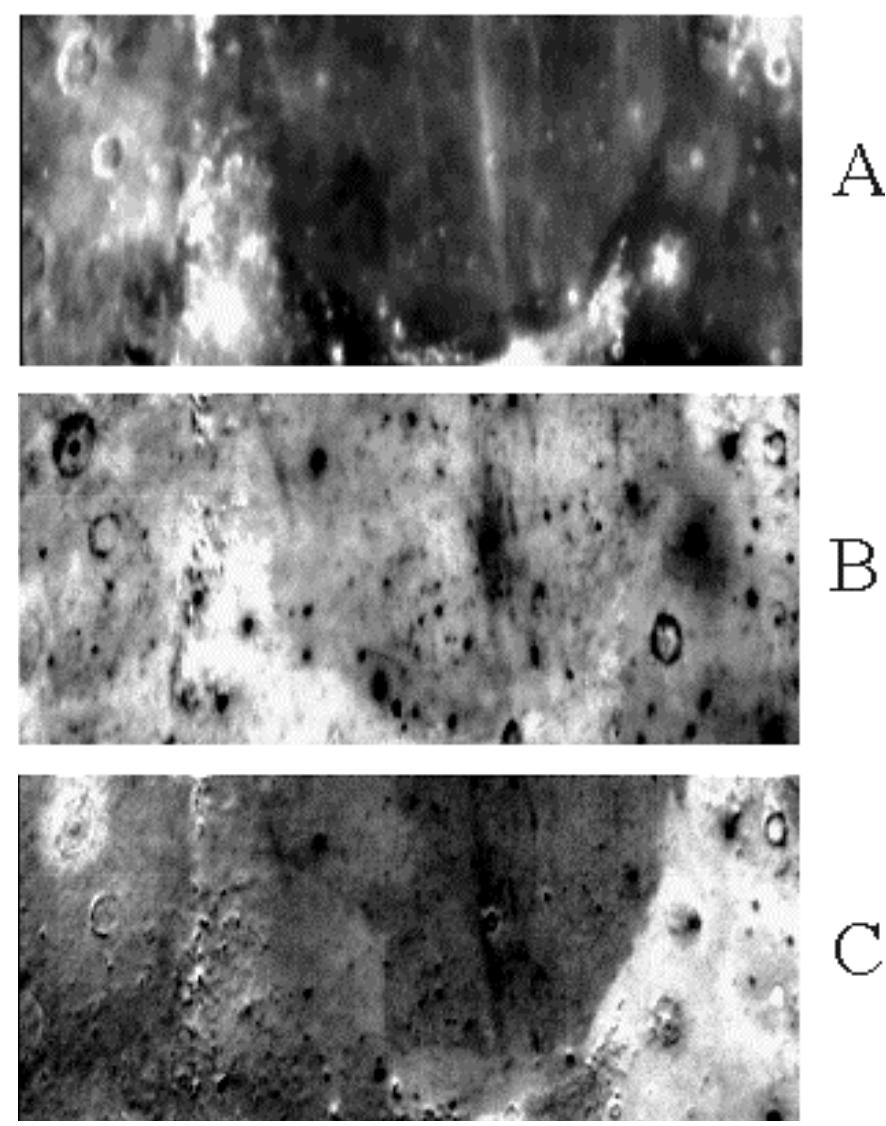

Fig. 3. Principal components of the image cube discussed in the text

Figure 2 shows a raw image at $0.7 \mu \mathrm{m}$ of the Mare Serenitatis/Tranquillitatis, Montes Haemus and Plinius region on the Moon. The scale is 1.2 arcsec per pixel, while the seeing is about 2.5 arcsec. The small crater in the center of Serenitatis is Bessel and will be used in the following to evaluate the enhancement process. Figure 3 shows the PC images. The PC1 image (Fig. 3a) is the albedo picture. In the PC2 image (Fig. $3 \mathrm{~b}$ ) the Menelaus rim and 


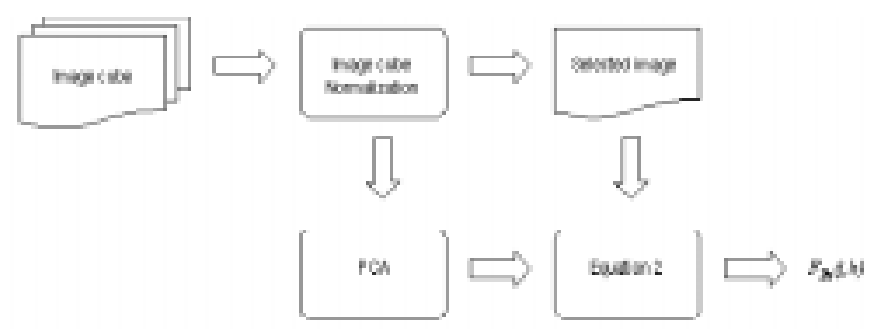

Fig. 4. Block scheme of the sharpening method described in the text

some features inside the crater are visible; also the $\mathrm{Al}-$ Bakri rim and floor are well discernible. In general, the PC2 image shows more details than raw image. The PC3 image (Fig. 3c) still contains enough information to allow recognition of narrow features as the Menelaus and Plinius rims and the Al-Bakri crater. Very narrow features, like small craters, crater rims and ejecta, are also well visible. In order to enhance the spatial contrast of the raw image shown in Fig. 2 by utilizing the results of the PCA, we have applied on the raw image $F_{\mathrm{R}}(j, k)$ the following transformation:

$$
\begin{aligned}
F_{\mathrm{En}}(j, k)= & \operatorname{stretch}\left[F_{\mathrm{R}}(j, k)\right] \\
& -\operatorname{stretch}\left[P C_{n}(j, k) \times L(m, n)\right]
\end{aligned}
$$

where $F_{\mathrm{En}}(j, k)$ is the enhanced image, $P C_{n}(j, k)$ is one of the three principal component images, $L(m, n)$ is a Laplacian convolution array. The $j$ and $k$ indices are the spatial coordinates of a pixel within the image, while $m=3$ and $n=3$ are the dimensions of the convolution array $L$. The Laplacian filter is:

$L(3,3)=\left[\begin{array}{rrr}0 & -1 & 0 \\ -1 & 4 & -1 \\ 0 & -1 & 0\end{array}\right]$.

It is a second derivative edge enhancement filter that operates without regard to edge direction (Ross 1995). In order to display the result when both positive and negative pixel values rise, a stretch operation is applied to each of the right-hand terms of Eq. (2). In this way, brighter and darker values produced by the Laplacian can be seen. The stretch $[I]$ is a linear scaling operation:

$\operatorname{stretch}[I]=\frac{I-\min (I)}{\max (I)-\min (I)} \times 255$.

Figure 4 shows schematically the sharpening procedure.

\section{Results}

The $F_{\text {En }}(j, k)$ images obtained through the relation (2) are shown in Fig. 5. It is possible to see how the images showing more spatial details are those obtained by using the second and the third principal component (Figs. 5b and $5 \mathrm{c}$ ). Figure $5 \mathrm{a}$ does not contain any spatial detail; this fact was quite expected because the PC1 image is
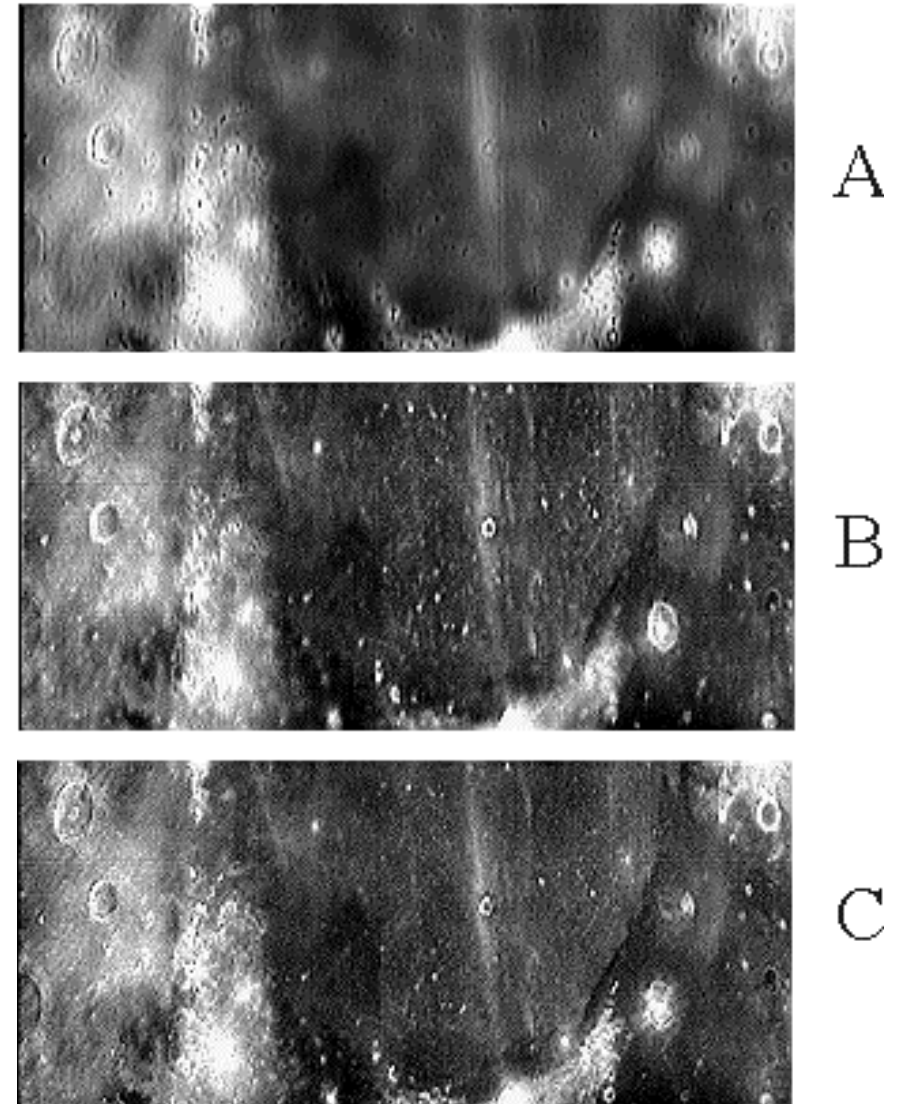

Fig. 5. Enhanced images obtained by means of relation (2). b-c) show more spatial details respect to the raw image shown in Fig. 2

not sensible to the spectral content of the data. In order to quantitatively evaluate the performances of the enhancement technique in terms of spatial details, in Fig. 6 horizontal profiles across Bessel and Dawes craters taken, respectively, on the $F_{\mathrm{R}}$ and $F_{\mathrm{En}}$ images are shown. Here, we have used $F_{\text {En }}$ obtained by means of the PC2 and PC3 images. Note the great increase of spatial contrast. The two peaks visible in the center-right plots correspond to the western and eastern rim of the two craters. The technique has spatially resolved the rims of the Bessel and Dawes craters, not visible in the raw image. The effect of the technique has been to remove the surrounding mare contribution from the compositional different craters rim.

\section{General remarks and conclusion}

The principal component analysis is very sensitive to spectral shape. For this reason, the spectrum must not present strong variations, as those due for example, to ice absorption bands occurring in the near infrared region, otherwise the result will be mainly driven by these spectral features. The most suitable spectral window for enhancement purposes is the visible because the spectra of planetary bodies present a smooth increase in reflectance 

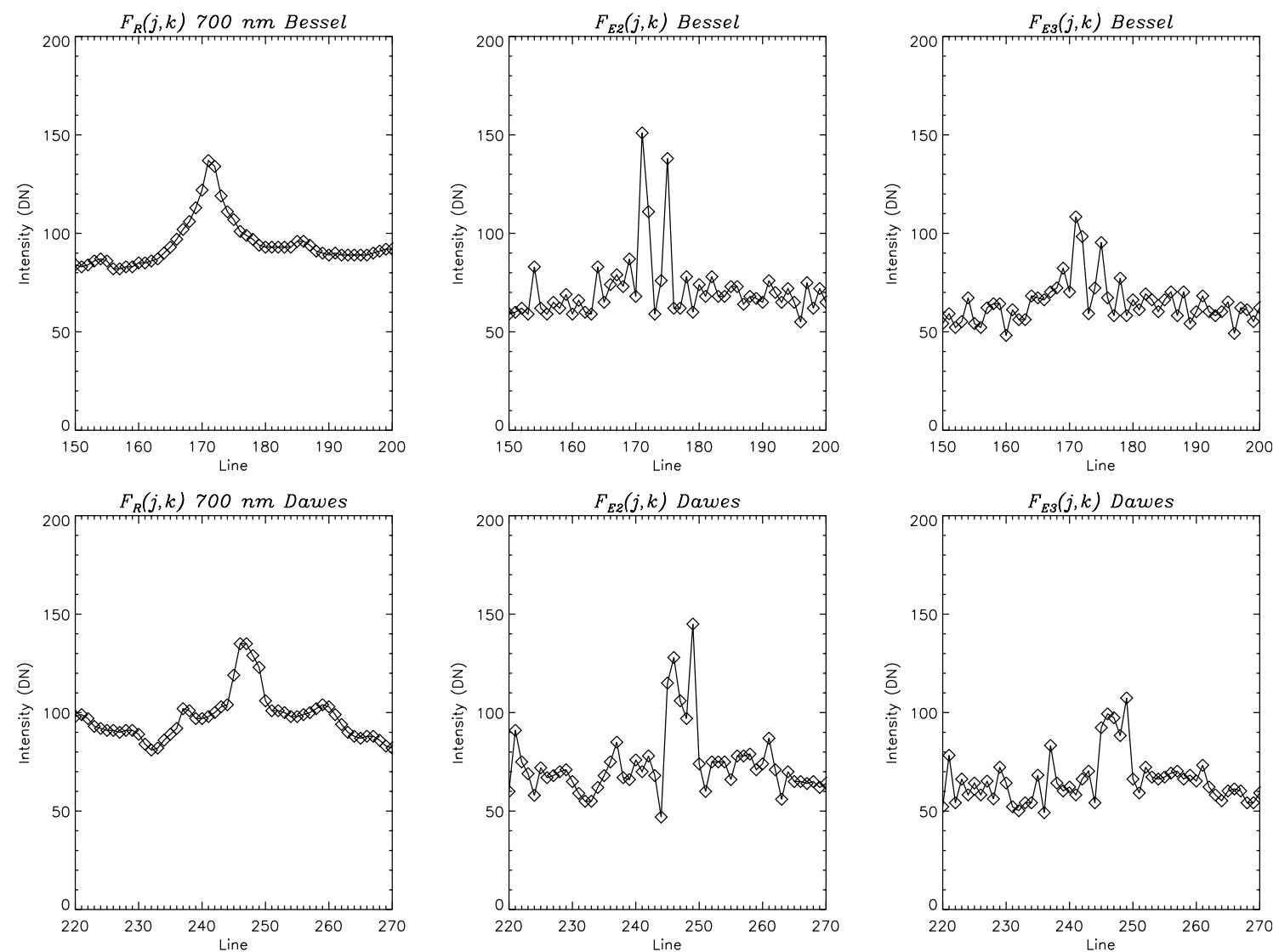

Fig. 6. Profiles across the Bessel and Dawes craters taken on the $0.7 \mu \mathrm{m}$ raw images (left panels) and the PC3 images (center and right panels). Note how the spatial contrast has been improved

toward longer wavelengths with some small absorption, generally close to $0.8-1 \mu \mathrm{m}$. Far from being complete, in the following we want to indicate a possible extension of the sharpening technique to space born imaging spectrometers. Unlike with ground-based telescopes, for which atmospheric seeing is the major source of image degradation, pointing errors are the dominant source for diffractionlimited telescopes in space. Pointing systems have a large number of random, and systematic errors. Cross track, along track, encoder errors, control law parameters (pole, zero for DC systems), gear periodicities, mount models (e.g., flexure, mirror sag/slip) and others (Brown 1993). Pointing errors distribute the light from a point source over a larger area and reduce the intensity of the image peak, which is to say its Strehl ratio. The Strehl ratio is the ratio of the intensity of the actual image peak to that of the theoretically perfect image for that system. An optical system is commonly called "diffraction limited" if its Strehl ratio is greater than 0.8. For example, for an optically perfect Hubble Space Telescope, the pointing errors should not be more than a few milliarcseconds in order to maintain diffraction limited quality in the ultraviolet (Brown 1993). Therefore, the technique illustrated above could also be used to mitigate the jitter effects on hyperspectral images. Although we have developed this method having in mind planetary applications, it could also be applied to CCD multispectral images (e.g. the classical $U$, $B, V, I$ filters). However, as already mentioned in the second section, the result should be less impressive, due to the small number of spectral bands compared to what is achievable with imaging spectroscopy.

Acknowledgements. We are particularly grateful to the support staff and telescope operators of the Sierra Nevada Observatory. Without their help the observations described in the paper would not have been possible. We specially thank Dr. J.L. Moreno and Dr. J. Rodriguez of Institute of Astrophysics of Andalucia for their continuous assistance. The help of Mrs. S. Zampieri and Mr. R. Perciballi in the manuscript preparation is also gratefully acknowledged. Funding were provided by ASI and CSIC grants.

\section{References}

Adams J.B., 1974, J. Geophys. Res. 79, 4829-4836 Adams J.B., 1986, J. Geophys. Res. 91, 8098-8112

Aptaker I.M., 1987, in: Vane G. (ed.), Imaging Spectroscopy II: Near-Infrared Mapping Spectrometer (NIMS) for investigation of Jupiter and its satellites, p. 196-212

Bellucci G., et al., 1998, Planet. Space Sci. 46, 377-390

Bibring J.-P., et al., 1989, Nat 341, 591-592 
Brown R.A., 1993, The Future of Space Imaging

Chevrel S.D., et al., 1994, Lunar Planet. Sci. Conf. 25th, 249

Davis J.C., 1986, Statistics and Data Analysis. In Geology. Wiley, New York

Erard S., 1991, Proc. Lunar Planet. Sci. Conf. 21st, 437-455

Fried D.L., 1966, J. Opt. Soc. Am. 56, 1372-1379

Goodman J.W., 1985, Statistical Optics. John Wiley \& Sons, New York

Hanish R.J., White R.L., 1993, in: Hanish R.J. and White R.L. (eds.) The restoration of HST images and spectra, Vol. II

Jaumann R., 1991, J. Geophys. Res. 96, 22, 793-22807
Juergens D.W., et al., 1990, International Journal of Imaging Systems and Technology 3, 108-120

Ross J.C., 1995, The Image Processing Handbook. 2nd edition CRC Press

Singer R.B., McCord T.B., 1979, Proc. Lunar Planet. Sci. Conf. 10th, 1835-1848

Smith M.O., et al., 1985, Proc. Lunar Planet. Sci. Conf. 15th, Part 2, J. Geophys. Res. 90, C797-C804

Vane G., et al., 1984, IEE Trans. Geosci. Remote Sens. 6, 546549 